\title{
Technology and Critical Cultural Understanding
}

\author{
Charalampos D. Kokkinos \\ Humanities Department, Hellenic Open University, Athens, Greece \\ Email: chared@central.ntua.gr
}

Received 20 February 2016; accepted 2 May 2016; published 5 May 2016

Copyright (C) 2016 by author and Scientific Research Publishing Inc.

This work is licensed under the Creative Commons Attribution International License (CC BY). http://creativecommons.org/licenses/by/4.0/

c) (i) Open Access

\begin{abstract}
In our everyday lives, we come into contact with a series of technological objects and we employ technology in a number of ways. Usually, the "relation" we develop with these objects works, on a first level, for our benefit. On the other hand, we actually know little about the technologies we use in order to accomplish various activities. Technologies have neither been developed, nor do they exist independently, even though we tend to perceive them as natural objects in themselves. Perhaps they are as much defined by causal laws, which are relevant to their "behavior" as specific artifacts, as they obtain ad hoc characteristics through our significations, which already belong to a specific social system. This ignorance of common sense often leads to the exclusion of a number of topics that are intertwined with the technological phenomenon from the everyday agenda of political debate. Moreover, the errors that stem from our unsophisticated or even unconscious attitude towards these artifacts have important consequences on various areas, including "development" and "work", education, the environment, and human communication itself. This paper will try to present elements of a critical theory of technology in order to illustrate the need to link the technological phenomenon with everyday political practice.
\end{abstract}

\section{Keywords}

Technical Rationality, Mediation, Critical Theory, Machine, Culture, Politics

\section{Introduction}

It goes without saying that the change we experience today, which is fuelled by a series of new technologies, differs from other profound changes that have defined our culture in the past. The current change affects our everyday lives, but the new tools it offers us can be seen as an extension of our senses, of our various modes of communication and, to a certain extent, of our brains (since the question about whether one regards machines as 
extensions of living organisms or living organisms as complex machines seems to be a topic of exploration as well). Nowadays, the proliferation of the fields of knowledge, the often vague distinction between art, technology and science, and the "immaterial" form of the new technologies compel us to widen the field of our traditional research disciplines, and most crucially the field of ethics. The debate around the morality of technology has given rise to special moral categories-regarding for example the issues of responsibility, safety and risk — which had not been as important in pre modern moral philosophy.

The starting point of this paper is the fact that what is at stake for the future of our own society includes, in its core, aspects of technology - seen as a whole and not ad hoc. We refer to technology as specific practices, artifacts and decision making processes and not as an intangible and abstract field that proceeds autonomously, deterministically and independently of the wider social context (Winner, 1986; Bijker et al., 1987; Feenberg, 2010a; Brey, 2010). In this light, we suggest that technology can only mean all the things that definitely partake (without being able to explain the reasons why) in the shaping of life and contribute in the status quo of a world that tries to discover ways and methods for its survival and development (Kokkinos, 2006).

Hitherto (and especially since the 1980s), several critical fields have been developed, but these have been centered only on specific manifestations of new technologies. What is missing, therefore, is a complete critical perspective of the technological phenomenon that will start from the irrefutable fact of the interconnection and interaction between various fields. This critique should be neither fragmentary nor disconnected from the wider social developments that take place in the context of political decision making. What are the undefined aspects of the basic characteristics of a "lifestyle" that has been mainly based on new technologies and what is meant when we refer to a "technological society?" How are innovative ideas connected with the end products and, more importantly, with the way they are incorporated in our everyday lives? Are our needs real (and are they satisfied by the utilization of human and natural resources) or are they artificial and are created in order to conceal the shortcomings of the political system?

In the present paper we will try to address only some of these questions within a concise framework of a critical approach to technology. Our goal is to lay down a reference framework for a potential exploration of the development of our social system based on a fuller investigation of the complexity of the "technological realm" regarding mainly the latter's relation to the current "state of affairs".

\section{Technology and Discontinuity}

Technologies seem disconnected from their past. We usually have no idea where they come from and how they were developed even though we tend to perceive them as natural objects in themselves. Perhaps they are as much defined by causal laws, which are relevant to their "behavior" as specific artifacts, as they obtain ad hoc characteristics through our significations. This ignorance of common sense often leads to the exclusion of a number of topics that are intertwined with the technological phenomenon from the everyday agenda of political debate. We are unaware of the conditions that shaped the decisions which defined their characteristics. They seem "self-sufficient" in the context of their rational functionality. A proper presentation and explanation of the functions of any device seems to be a constitutive part of the process of researching and discovering the causal links of their parts. Yet, it is also true that no device came about suddenly and in complete form based only on the criterion of functionality. Every developmental process is full of accidents, choices and alternatives. The perfection of a technical object erases the traces of the labour needed for its creation, as well as the traces of the social forces that were in play during the period of the finalization of its design. This process allows the object to accommodate itself in its environment and therefore the exclusion of its past contributes to the oblivion of the set in which it belongs (Feenberg, 2010a).

In everyday life, it is not, in principle, necessary to know the laws that govern the construction and function of things. We can use electricity even without knowing its laws and this stance is no different than the attitude we had in the past, when we were able to drive a cart without knowing the mechanical laws that governed the function of the wheel. The effect of the (ambiguous) technical progress in our lives is that we need to learn how to use a growing number of things, while at the same time our spare time is diminishing. Moreover, our inability to produce the things we use ourselves is deepening. This is the consequence of the specialization introduced by technology. Without a doubt, in the relation between human beings and nature, our "freedom" depends on our ability to produce. But this is true for humanity as a whole, not for the individual. One of the great changes of our time is the short life span of an object. 
In traditional societies (and up until the dawn of the modern era), the whole of the human creative and productive energy — either they made shoes or built a cathedral — was focused on the maximization of the product's durability. Whatever they made had to endure for a long time. Today, the economy of permanence gives its place to the economy of transition (Kokkinos, 2006). Replacing a thing is often cheaper than repairing it. The development of technology has allowed us to create better products. As change gains pace and reaches the most distant places of the society, the uncertainty about future needs grows as well. By avoiding commitment to rigid forms and functions, we create products for short term use. As change becomes faster and complexity grows, it should be expected that the principle of one-use products will expand and the relation between human beings and things will decline even more. The question that arises is how necessary and effective are these new technologies.

According to our common sense, technologies succeed because they do their job well. Effectiveness is the measure of their value and explains why those specific decisions were made, instead of various other alternative options. Yet, the history of technology tells a different story. Frequently, in the first stages of a process of developing a product, no choice functions very well according to later standards; that is, when one of those choices have gone through many phases of innovation and improvement. When we look back, from the standpoint of an advanced device, we miss this point because of its apparent superiority and success. But this superiority is an effect of the original choice that favoured the creation of a successful technology against a whole array of alternative choices, and not vice versa. What, then, explains the specific choice? The social actors who make these choices employ various criteria. Sometimes, financial considerations prevail. In other cases, technical criteria are used, such as the accommodation to its environment, which contains various other technologies. A number of social or political demands are also at play. In other words, there is no universal rule that could explain the paths followed in the developmental process. Of course, all technologies must be more or less effective, but this does not explain why these technologies are present in our technological environment (Feenberg, 2002). In any case, only a study of the possible conditions for the success or failure of a device can reveal the whole truth.

\section{The Culturally Reformed Environment through the "Predominance" of Technology}

In technique, Adorno recognizes an essential role in the formation of the social reality and in the "reading" of artistic creation. "Artworks are enigmatic in that they are the physiognomy of an objective spirit that is never transparent to itself in the moment in which it appears" (Adorno, 1997 [1970]). At the same time, human beings create art through technique; in this way technique has a constitutive meaning for art. Moreover, although no work of art is just the sum of its technical elements, for Adorno it is technique that is the key factor in deciphering art, leading us to the core of the works of art.

An essential presupposition of thought, and thus of philosophy, is its emancipation from the object. However, nowadays the role of the various technological achievements, that must be complementary in all social activities, tends to become central. The thinking subject submits itself to the machine by admitting that the only way to fulfill his/her many needs is by using the repeated mechanical operation. The irrational structure of society which goes along with its "evolution" does not differentiate the dynamics of human thought from the mechanical way of production. In this context, many edifices transformed in the course of history into art, while previously they were not art, and others, that used to be art, cease to be. The subject of reality is not identical to itself, and non empirical, but it changes through history. It is the social environment which determines its position in the value scale of each society. Today we speak of works of art even when we refer to fractal objects (Kokkinos, 2013a). On the other hand, the difference between works of art and the empirical world, the character of their appearance, is formed in the relation they have with reality and, according to its tendency to resist reality, against it. Thus, if the artist would like to eliminate his/her reference to reality, he/she would abolish the premise of artistic creation per se (Adorno, 1997 [1970]).

In Aesthetic Theory, Adorno refers to the "parody of aesthetic semblance" (Adorno, 1997 [1970]), an issue that is interwoven with the notion of "cultural industry". The value of many products, through mass production, has become dubious and is replaced by the subordinate enjoyment of a kind of prestige to the consumer, i.e. to the enjoyment of the product as a commodity per se. The product of technique, the technological object, is presented today as the outcome of a "normal" process. However, in many cases and in order to create a product, illegal and manipulating means have been used. The domination of the "civilized" world against the majority of the society tends to become rational, to seem as the only solution for the overwhelming impasses of the West 
(the sole part of the planet, according to many people). In this context, aesthetic issues are reduced in the hierarchy of common action because there are more emergent needs to be satisfied. Modern age moves and exploits this same mechanism by ignoring, aphoristically, the tradition and the human-made heritage of objects. Tradition is not to be generally and abstractly rejected, but it must be criticized in a non simplifying way and always in accordance with the standards of the present. Nothing must be adopted uncritically, just because it exists and it used to be valuable, and nothing is outdated just because it is the byproduct of another age. "[T]ime alone provides no criterion" (Adorno, 1997 [1970]). At the same time, the New product seems to be the aesthetic feature of broad reproduction, along with its promise for indefinite abundance. In a society where the creative spirit's diachronism is disputed, and where every reaction against art seems confused, art breaks into reified cultural heritage and into hedonism which consumer gets from the continuously changing New object. People nowadays does not have the time to become acquainted with his/her creation, which is disdained by a new creation, and constantly plans or expects the replacement of an object by another one that does not differ essentially from the previous one. Social reality precludes the category of the older constructions, since there is no time for the "dialectical establishment” of any object. The ephemeral and without any aesthetic refinement product determines the modern way of life (Adorno, 1973 [1964]).

In view of its contribution to the notion of the Beautiful, Adorno's claims about the constructed environment are essential. According to Adorno, the internal changes that this notion underwent in nature are reflected in the fact that only in the course of the nineteenth century this concept was enlarged by a new domain: the cultural landscape (Kulturlandschaft), an artifactitious domain that must at first seem totally opposed to natural beauty. Historical patterns usually connected to their geographical environment with which they have some resemblances due to the same construction material (i.e. stone), are considered to be beautiful. Such patterns are not based, like the works of art, on a central morphological law, since they are barely planned as a whole. However, historical patterns are not intact, a quality that common sense attributes to the notion of beautiful in nature. Culturally reformed landscapes reflect history through their expression. Historical continuity gives them a certain form and unifies them dynamically. And, as Adorno states, “...without historical remembrance there would be no beauty" (Adorno, 1973 [1964]).

For Marcuse, the society that seeks the transformation of nature, which is achieved today especially by means of technology, alters the basic principles of domination, which changes its form. Thus, society replaces the personal dependence (the slave's dependence on the master, the bond slaves' dependence on the atheling, the nobleman's on the king) by a form of dependence of an "objective world order" (economic laws, the market etc.). In this context, technology becomes the main agent of objectification (Marcuse, 1971 [1964]), since technological thought enters into nonmaterial, mental activities. In this way, power seems to lose its exploitive and oppressive character and becomes "rational". The criterion of the rationalism of power is the preservation of a system that bases its legitimacy on scientific/technical progress.

Marcuse's contribution as to the discussion of mass culture and aesthetic theory is crucial. In Eros and Civilization (Marcuse, 1981 [1955]) he speaks in a total, aphoristic way for the necessity of "great art" that can contribute to the establishment of a different kind of society. In Counter-revolution and Revolt (Marcuse, 1974 [1972]) and especially in his latter work, Aesthetic Dimension (Marcuse, 1998 [1978]), Marcuse, referring to the necessity of a radical political action that shall transform the wretched reality, summarizes his problematization in favor of the precedence of aesthetics. He does not hesitate to argue that art questions the monopoly of established reality to determine what is real, by formulating a phenomenal world which however is more real than reality itself. Art, for Marcuse, has its own language and reveals reality only by means of this language. It has its own dimension of affirmation and negation, a dimension that cannot be synchronized with the dimension of mass production. All authentic works of art are revolutionary, i.e. subversive; they criticize the established reality and reveal the image of emancipation. The aesthetic form and the internal coherence of artistic creation constitute the dimensions of truth, criticism and the promise of art. That means that, while art is inevitably a part of what exists, and it criticizes what exists only as a part of it, at the same time this contradiction is preserved and overcome through the aesthetic form, which gives the power of alienation into the everyday experience. Thus, a new consciousness and a new conception arise in the context of reality, a form of which is art.

An object functions in an aesthetically way when it causes tensions, when it communicates with the limits of the senses and those of cultural data and patterns, when it offers a version of reality and when it doesn't recognize preconfigured intellectual patterns. In order to be accepted, the aesthetic object, either a result of personal or collective action, being singular or plural, it presupposes certain conditions in various levels of social and 
cultural life, conditions that stress the importance of human participation in the process of its production. Today this participation declines; it is reduced to the passive management of mechanical functions towards a result that is known in advance. The multiplicity of artifacts, the "democratization" of material culture by means of technical reproduction, made works of art available to everyone. However, mass production has reversed the main preconditions of artistic creation. The authentic is no more an object of admiration, a source of inspiration and awe. Now there exists the copy of which quality (its relation to the original) constantly improves. The originality of an artifact depends in something more than the sum of its elements: from its mechanical strength to its value as historical evidence. Since the latter is based on the former, through the predominance of the copy and through the cancellation of authenticity, the value of historical evidence is also postponed. As Benjamin characteristically mentions, what decays in the era of technological reproduction of the work of art is its aura (Benjamin, 1978 [1936]).

The technique of reproduction, as opposed to the preservation of the original, detaches the product from tradition, because this uniqueness of the work of art is to be identified in its incorporation in the web of the latter. Constantly increasing the number of copies, the new "technological state" replaces the one and unified presence with mass domination, it enhances consumption in a world that could not be based solely on the laws of industrial mass production (Kokkinos, 2013b).

\section{Machines and Computers: Cultural Interpretations}

The meaning of the term machine has shifted, from the ancient manually-operated instrument of work, to the automatic tool. It is noteworthy that the noun machine and the adjective mechanical derive from the Latin word machine and the Greek word $\mu \eta \chi \alpha v \eta$ correspondingly, while the related Greek verb $\mu \eta \chi \alpha v \varepsilon v ́ o \mu \alpha \iota$ means "to manufacture, to invent or to machinate". Going further back in time, the word machine seems to relate to the Indo-European roots mogh- and megh-, and thus to the German root maxan (whence the German words Macht and machen), meaning "to have the power to" and thus connected to the English verb may (Mitcham, 2005 [1994]). Since pre-modern mechanical power stems from the human body and is manually distributed, the adjective mechanical kept its close connections with manual work in Latin and English until the $17^{\text {th }}$ century. But along with the development of non-manual sources of power in modernity, there has been a shift in the meaning both of the noun and the adjective. Thus, from a historical point of view, the word machine has denoted three different things (which may all be compatible with the definition of machine as an instrument to transfer strength or to transform its implementation): first, machine may refer to the simple machines of Antiquity, such as the lever, the wedge, the wheel and the axis, the pulley, the screw and the inclined plane. Second, a machine may be any tool or simple machine of a certain magnitude, requiring several persons to operate it, due to its power requirements. Finally, a machine may be a tool not dependent upon human force, though requiring some kind of human control or guidance (in the case of a car, a person controls the car, while the engine makes the car move).

As has often been stressed, the difference between the tool and the machine has never been accurately defined. Yet the common perception is that a tool is a manually-operated machine, while the term machine denotes an instrument, or an aspect of an instrument, independent from human intervention. Moreover, one should note the common reluctance to ascribe the name of "machine" to an automated device designed and made (or at least assembled) by human actors, but not directly activated or guided by humans. Thus, as the machine tends to become increasingly independent from the input of human force, its character as an object radically changes; it becomes not a simple static object, but a vehicle and a producer of functions or particular physical, chemical or electrical processes. The most important shift is of course the shift from the tool to the machine. The steamengine and the internal combustion engine are not just objects, like hammers or saws. The gasoline inside an internal combustion engine performs a specific function which is made possible only under the conditions set by the machine itself. The design and the construction of such machines of production and integration of processes entail the construction not only of a physical object but also of a process. What happens inside an electrical converter or inside an internal combustion engine does not happen outside of it. The process of electricity production or of internal combustion takes place only under human-determined conditions, which is what makes it artificial. The more machines become "independent" from the input of human force, in a spectrum covering mechanical, chemical and electrical processes, and subsequently are assembled in systems, the more they become increasingly objectified processes (Giedion, 1969; Adas, 1989; Jennings, 1995; Kasson, 1999). 
Historically, the machine as process has evolved from the converters of water power to mass production (first of textiles and then of chemicals), to processes of mechanization and assembling (through mechanical tools) and to industrial production lines. For example, there is a difference between the use of tools or even machines in order to produce a number of separate and distinct artifacts, even when such artifacts are more or less identical, and the mass production of a completely homogenous and uniform product, prepared in separate lots or even serially. Indeed, the very term product tends to prove this distinction. In any case, the machine or the object that makes possible such processes must also be viewed as a process. Such an object, integrated in a technical system, becomes less and less an "entity" capable of developing an infinite number of different and distinct relations, and more and more a "moment" within a system of predetermined relations. Typical examples here might be the systems or networks of computers, to which this paper now turns.

The dominant cultural interpretation of artifacts considers tools and machines as physical extensions of human body. If tools (or machines in the classic sense) had the ability to enhance human force, they did it just by magnifying the inherent human force. If machines in the second sense (as tools operated by several humans) did the same, they did it just by uniting the inherent human forces of the members of the team. Contemporary energy machines have this effect in a different way, by putting non-human forces at the disposal of individual human guidance. Thus, while tools are instruments of a function separating, specializing, distributing or concentrating the total force of the human body, machines, by contrast, integrate the human hand as a link of organs in the multifarious acts of a person guiding or using non-manual forces.

Contemporary machines, unlike traditional tools, include a qualitative distinct separation of the human agent from his or her body and the primitive body experience. A mechanical work in the pre-modern sense concerns a work performed manually and is as such dependent upon human force. This work does not have anything to do with superior, superhuman or spiritual forces, but remains purely physical. By contrast, mechanical work in its modern use is a work performed without special attention, in a repetitive way. Modern machines alienate the human agent from the sensory and motive mind-body complex. For this reason, in a contemporary view, a regression to primitive mechanical processes could be considered as a desirable development which would reunite the mind with the body. In this context, Mumford and others have argued that "the specialized user of tools becomes increasingly accurate and automated, mechanical, in a word, as his initially purposeful moves are crystallized into simple reflexes” (Mumford, 1963). Such generalizations rest upon a minimal experience with tools. When a function becomes mechanical, machine-like, human control over it tends to decline. Admittedly, accuracy, in the sense of faithfulness to an imposed, geometrical line, is better served by electrical tools, but in this way a certain response to materials tends to decline. No machine can be compared to the human hand. Machines offer speed, power, full uniformity and accuracy, but no creativity, adaptability, freedom and differentiation. This argument is confirmed by studies about the formation of skills and cases of positive and negative transfer of partial skills (Bruner, 1973). A person developing a tendency to respond to a generic work in a predetermined way acquires a formed skill. Although certainly useful, such a skill may cause the development of a kind of inability to optimally respond to a variation of the work in question. For example, an electric saw may easily get out of control, while a wound caused by a manual saw is normally less serious than a wound caused by an electric saw. It is no accident that the termination of a work or delicate artistic work is still done manually. Maybe this automated, uncritically repeated process characterizing work in modernity is clearer in the field of computer science.

Contemporary technological developments were based on the revolution of computer science. Computer science was closely connected with the development of a particular machine, the computer. Computers are the major achievement of the $20^{\text {th }}$ century, although the first steps were taken much earlier, with Pascal's adding machine, Leibniz's four-operation machine and Babbage's ambitious mechanical projects, which foresaw in a certain sense the contemporary achievements. But the theoretical grounds of contemporary computers were set by Turing. Turing was the first to clearly see the vast potentialities of computers even before their creation and he also investigated the question of artificial intelligence, which is now the most advanced field of theoretical and applied computer science. Based on Turing's theoretical model, Hungarian-American mathematician Von Neumann and his associates formulated the solution that led to the manufacture of the contemporary computer machine ten years later. The most important thing about this "revolution" is that it does not require expensive materials and material capital accumulation, as was the case with the $18^{\text {th }}$ century Industrial Revolution, since the raw material for the production of computers is silicon, an element found in abundance all over the planet. The driving force of computers and computer science is the human brain. The industrial cost of the semiconductor 
microchips is about $80 \%$ theoretical and applied research, $17 \%$ executive work and just $3 \%$ raw material.

Closely connected with computer science, Cybernetics has been defined as "the science of all possible machines" (Wiener, 1970 [1948]). As a theory of the ways in which the various situations of information interact in order to produce particular types of behavior, cybernetics interprets the nature of technology in terms of information processing and proposes a way to guide and control this processing. The foundation of the determinable behavior in cybernetic theory is the technical concept of information. Information is described as a predetermination of the possibility to manifest a kind of behavior. In classical mechanics, a machine is a configuration of mechanical parts in which any input of energy into the system is designed to cause certain predetermined moves with the least possible energy loss (as a result of the various resistances). A cybernetic device is a communicative configuration in which any input of information into the system is designed to cause certain predetermined information outputs with the least possible information loss. A machine can no longer be considered as a closed chain of motions, but may be reconsidered as a closed configuration of information. The most advanced cybernetic devices, the so-called "smart" ones ("smart" buildings, transport, home devices, etc.), are functionally auto-regulated. Wiener defines cybernetics as the science "of all possible machines", whether electronic, mechanical, neural or economic in nature. During its early development, in the 1940s, it was closely related to neurophysiology, as a result of the assumption that the apparatuses of negative feedback are of central importance in the central nervous system. As a general theory of artifacts and functions (from corrective neurosurgery to business management), cybernetics proposes a radical extension of the scope and the purposes of technological knowledge. Indeed, the general view of reality in cybernetics shows many features of a technological philosophy or even an engineering philosophy of technology. In traditional theory, the difference between living and inanimate beings was that living beings are characterized by self-motivation, unlike the inanimate beings. An aspect of this character of living beings is that they either possess an inner energy source or are able to gather the energy that they need for their motion from external sources of the broader universe, by acting on their own initiative. Despite their apparent automotive character, machines cannot provide "themselves" the energy required for their motion. Classical modern technology is a technology of power, focused on ways of production and transmission of energy. In cybernetics, emphasis is given not to sources of energy but to determined functions; the availability of energy is taken for granted. Cybernetics is the science of all forms of behavior insofar as they are predeterminable or reproducible. Since both human beings and machines show a regularity of behavior, cybernetics rejects the traditional distinctions between humans and machines, living beings and inanimate objects, thereby raising a series of ethical questions.

At first, ethical questions concerning computers were raised in light of threats against privacy and business security, as well as violations of digital databases and networks. Such questions are related to concerns about the ethical codes of the computer science professionals, responsibility for software misuse, copyrights and the relation of computers and social power. In a more general way, computer "ethics" poses questions about the use and structure of information, no less within cybernetics. For example, what are the ethical guidelines for the creation, diffusion and use of information, not just in the case of computers, but also in any kind of information management media, from telephone and radio to television and satellites? Moreover, is it not possible for some software programs to exceed human responsibility because of their inherent complexity and incomprehensibility? Is it not true that some forms of information technology are so complex that even their own creators are unable to comprehend or control them? In this light, it is perhaps necessary to develop an ethics of information in some cases, probably quite similar to medical ethics. Even if the reliability of complex information systems could be secured by advanced techniques, there would still be an open ethical question concerning the right relation to the virtual reality created by such artifacts, as well as concerning the reified decision-making principles or processes. Other questions of this kind fuel a broader reflection on technology, science and engineering (Tassios, 1984; Scharff \& Dusek, 2003; Kokkinos, 2012).

\section{The Politics of Technology}

At a first glance, it seems that two separate things—-technical knowledge and everyday experience-interact in a "battle" of opposites. Experts sometimes claim that we should not allow values and desires to obscure facts and scientific truths. Those who complain possibly commit the same error when they vaguely attack the experts, while at the same time continue to use technology in their everyday lives. In fact, technical knowledge and experience are complementary and not conflicting fields. Technical knowledge is incomplete without the facts of 
experience, which are used for the correction of its shortcomings and its simplifications. Popular complaints indirectly reveal the accidental complications that are caused by those simplifications and, thus, they bring to the fore aspects of nature that were neglected by the experts. Complaints bring about values and priorities. The need for such things as safety, health, specialized work, recreation grounds and tasteful cities confirm the failure of technology to adequately incorporate all the restrictions of its environment. Inevitably, such values will be incorporated in improved technical designs and the conflict between the public and the experts, in some cases at least, will subside. But values cannot be incorporated into technology unless they are translated into technical language. Reality in itself does not stand on the one side as an object, independent, pure, given, neutral and indifferent and accordingly, the human mind is not self-contained and self-reliant, as an acting subject, which is always ready to take advantage of the nakedness of reality in order to penetrate it. Exploring the concept of the artifact, we can see the "socialization" of the objects that gives them a unique "nature" (Kokkinos, 2012). Wishful thinking is not enough if we want to eliminate relevant technical restrictions. In order to make something useful with public interventions, experts need to find a way to express values as sustainable technical specifications. When they do so, a new version of the contested technology can be produced; and this version will be suitable for its environment. During this process, values are translated into technical facts and technology is incorporated into its environment in a more harmonious way. Values are not the opposite of facts; they are not subjective desires, unrelated to reality. Values express aspects of reality that have not yet been incorporated in a given technical environment. This environment has been shaped by the values that prevailed during its creation. Technologies are a fixed expression of these values. New values lead to the revision of established designs.

Social groups are constructed around technologies that mediate the relationships between their members, allow the emergence of a common identity and shape their experiences. We all belong to such groups. Some of them are established social categories and it is more than obvious what part technology has played in the shaping of their experience. Consumers and victims of the side-effects of technology are latent groups that emerge when their members realize that the causes of their problems are common. The politics of technology develops through such technical mediations, which are the foundations of the various social groups that comprise a society. Such relations between individuals and the technologies that connect them cause varied effects. Social identities and worlds emerge, shaping the backbone of a modern society. Once they establish themselves and become aware of their identity, technologically mediated groups influence the technological planning through their choices. This feedback from society to technology is, according to Feenberg, the democratic paradox: "the public is constituted by the technologies that bind it together but in turn it transforms the technologies that constitute it” (Feenberg, 2010b). Neither society nor technology can be perceived separately from one another, because neither of them has a fixed identity or form. Those who demand an environmentally compatible production, a healthcare system that corresponds more to the needs of the patients, a free and public Internet, and various other democratic reforms of technology, expand their democratic demands in order to cover the social field that is incorporated in the technological system.

\section{Coda}

In modern society, technologies are considered purely instrumental, cut off from their past, the environment in which they operate and their user. Such reasonable distinctions hide essential aspects of technology and create an illusion. This illusion is not a fundamental problem in traditional societies. In these societies the knowledge of traditional handcraft and everyday experience are in constant communication. Any new knowledge that comes from the use of technical devices is absorbed by the handcraft tradition. Technical activity is restricted and controlled in this context. From the viewpoint of the modern society, this seems to be an obstacle for the development, but on the other hand, this restriction might have its own wisdom. Without a doubt, our experiences with technologies like nuclear weapons and toxic chemicals show that there is a need to introduce limits and restrictions. In modern times, the control of technology is no longer in the hands of craftsmen, but of business owners. A business is different because it has both a very limited goal—profit—and the liberty to accomplish this goal without taking the consequences under consideration. Since technology has been introduced into such an institution, the lessons of experience are ignored. Technological development can move on, without considering the most distant areas of its own framework. This leads to the creation of complex and sophisticated technical fields of rapid "progress", but with miserable outcomes. Instead of trying to interpret and correct the technological illusion, modern societies consider it to be real. They imagine that they can operate in the world 
without any consequences for themselves. Nevertheless, human activity, including technical activity, unveils the actor.

The character and goals of technology do not constitute an inherent problem of technology itself. For its aims and directions, for the meaning of these aims, for the conditions and the consequences of its development, we need to construct a set of values, according to which the technological phenomenon can develop in a humane manner. This is a task for us all. In order to assume control of technology and in this way to influence its rapid growth, we need to start subjecting new technology to strict tests before we unleash it. Before we evaluate an innovation, we should ponder about a series of novel problems that are connected to it, as well as about its longterm consequences on the social and cultural environment. Additionally, we should ask in what way the proposed technology will affect our social value system-apart from the changes it will cause to the social structure.

Nowadays, technological issues appear regularly on the front pages of newspapers. There is a chance for a radical change in the way we perceive technology. Technology is increasingly put in the foreground of our everyday activities, leading to a revival of philosophic thought.

\section{Acknowledgements}

The author would like to express his gratitude to two anonymous reviewers whose valuable and constructive suggestions helped improve and clarify the manuscript.

\section{References}

Adas, M. (1989). Machines as the Measure of Men. Ithaca and London: Cornell University Press.

Adorno, T. (1973 [1964]). The Jargon of Authenticity. Evanston, IL: Northwestern University Press.

Adorno, T. (1997 [1970]) Aesthetic Theory. London: Athlone.

Benjamin, W. (1978 [1936]). Art Issues. Athens: Kalvos.

Bijker, W., Hughes, T., \& Pinch, T. (Eds.) (1987). The Social Construction of Technological Systems. Cambridge, MA: MIT Press.

Brey, P. (2010). Philosophy of Technology after the Empirical Turn. Techné: Research in Philosophy and Technology, 14, 36-48. http://dx.doi.org/10.5840/techne20101416

Bruner, J. S. (1973). Beyond the Information Given. New York: W.W. Norton.

Feenberg, A. (2002). Transforming Technology. A Critical Theory Revisited. New York \& Oxford: Oxford University Press.

Feenberg, A. (2010a). Between Reason and Experience: Essays in Technology and Modernity. Cambridge, MA: MIT Press.

Feenberg, A. (2010b). Ten Paradoxes of Technology. Techné: Research in Philosophy and Technology, 14, 3-15. http://dx.doi.org/10.5840/techne20101412

Giedion, S. (1969). Mechanization Takes Command. New York: W. W. Norton.

Jennings, H. (1995). Pandaemonium. The Coming of the Machine as Seen by Contemporary Observers. London: Papermac.

Kasson, J. (1999). Civilizing the Machine. New York: Hill and Wang.

Kokkinos, C. (2006). The "Predominance” of Technology and the "Expansion” of Culture: Outline of a Theory for the Interpretation of Technological Civilization. Deucalion, 24, 273-295.

Kokkinos, C. (2012). From Technology to Engineering: On the Epistemological Status of the Concept of the Artifact. International Journal of Terraspace Science and Engineering, 5, 125-137.

Kokkinos, C. (2013a). The Signification of Objects in the Context of a Critical Examination of Technological Civilization: An Interdisciplinary Approach. International Journal of Humanities and Social Science, 3, 57-65.

Kokkinos, C. (2013b). Technology and Contemporary Human Condition: Cultural Expansion and Technological Intervention through Politics? Synesis, 4, 54-61.

Marcuse, H. (1971 [1964]). One Dimensional Man. Athens: Papazisis.

Marcuse, H. (1974 [1972]). Counter-Revolution and Revolt. Athens: Papazisis.

Marcuse, H. (1981 [1955]). Eros and Civilization. Athens: Kalvos.

Marcuse, H. (1998 [1978]). The Aesthetic Dimension. Thessaloniki: Nisides.

Mitcham, C. (2005 [1994]). Thinking through Technology. The Path between Engineering and Philosophy. Athens: NTUA Press.

Mumford, L. (1963). Technics and Civilization. San Diego, CA and New York: Harvest/HBJ. 
Scharff, R., \& Dusek, V. (Eds.) (2003). Philosophy of Technology. The Technological Condition. Oxford: Blackwell Publishing.

Tassios, T. (1984). Technocratic or Archimedean Engineering? European Journal of Engineering Education, 9, 29-34.

Wiener, N. (1970 [1948]). Cybernetics and Society. Athens: Papazisis.

Winner, L. (1986). The Whale and the Reactor: A Search for Limits in an Age of High Technology. Chicago, IL: University of Chicago Press. 